\author{
Diogo Müller de Oliveira, Diego Paulo Ongaratto, Luiz Antonio Mazzini Fontoura*, Fabrício Fredo Naciuk, Vinícius \\ Oliveira Batista dos Santos, Jéssica Danieli Kunz e Marcelo Volpatto Marques \\ Departamento de Engenharia de Processos, Fundação de Ciência e Tecnologia, Rua Washington Luiz, 675, 90010-460 Porto Alegre \\ - RS / Curso de Química, Universidade Luterana do Brasil, Rua Farroupilha, 8001, 92425-900 Canoas - RS, Brasil \\ Alexander Ossanes de Souza e Claudio Martin Pereira de Pereira \\ Centro de Ciências Químicas, Farmacêuticas e de Alimentos, Universidade Federal de Pelotas, Campus Universitário, Capão do \\ Leão, s/n, 96010-900 Pelotas - RS, Brasil
}

Dimitrios Samios

Departamento de Físico-Química, Instituto de Química, Universidade Federal do Rio Grande do Sul, Av. Bento Gonçalves, 9500 , 91501-970 Porto Alegre - RS, Brasil

Recebido em 10/9/12; aceito em 16/11/12; publicado na web em 6/3/13

\begin{abstract}
TRANSESTERIFICATION DOUBLE STEP PROCESS FOR BIODIESEL PREPARATION AND ITS CHROMATOGRAPHIC CHARACTERIZATION: OILS AND FATS IN PRACTICAL ORGANIC CHEMISTRY. Methanolic transesterification of oils and fats was carried out in a two steps procedure, under basic and acidic catalysis. Palm, soybean, canola, corn, rice, grapeseed, sunflower, peanut, pequi and olive oils, besides tallow and lard were used as feedstock. Specific gravity, relative viscosity, thin layer chromatography and gas chromatography were used to characterize the biodiesel. Biodiesel was obtained in high yield and purity. Results were used to discuss the following key-concepts: 1 - triglycerides, composition and properties; 2 - nucleophilic acyl substitution under basic and acid conditions, 3 - thin layer chromatography, 4 - gas chromatography and its quantitative methods.
\end{abstract}

Keywords: biodiesel; chromatography; oils and fats.

\section{INTRODUÇÃO}

O biodiesel, hoje, assim como outros biocombustíveis, é uma realidade e sua produção e consumo vem crescendo no mundo inteiro. No Brasil, sua adição ao diesel fóssil tornou-se obrigatória em 2008. Hoje, é usado na proporção de 5\% no combustível comercializado no país. Informações sobre este assunto são veiculadas constantemente em todos os meios de comunicação. Como tema de estudo, o biodiesel oferece oportunidade de contextualizar um largo espectro de tópicos em diferentes áreas, incluindo energia, meio ambiente, tecnologia química, análise e síntese orgânica, apenas como exemplos.

O biodiesel é constituído por uma mistura de ésteres obtidos por esterificação de ácidos graxos ou por transesterificação de triglicerídeos, Esquema 1. Sua principal aplicação é como combustível, podendo substituir parcial ou totalmente o diesel em motores com ignição por compressão. ${ }^{1-7}$

Como combustível, o biodiesel apresenta diversas características vantajosas quando comparado com o diesel. Do ponto de vista ambiental, é livre de compostos de enxofre e aromáticos, tem uma menor contribuição no efeito estufa, é biodegradável e obtido a partir de fontes renováveis. Com relação aos aspectos econômicos, pode diminuir a dependência dos países importadores de petróleo daqueles que o produzem e estimular a produção agrícola e o desenvolvimento rural. No que diz respeito ao desempenho, o biodiesel apresenta maiores ponto de fulgor, número de cetano e poder lubrificante. Por fim, pode ser usado em motores com ignição por compressão sem que haja a necessidade de modificações mecânicas. ${ }^{1-7}$ Evidentemente, há algumas desvantagens. O poder calorífico, isto é, a quantidade de energia gerada na queima por unidade de massa do combustível, é menor do que no diesel. Além disso, degrada-se por oxidação com facilidade e

*e-mail: lmazzini@uol.com.br

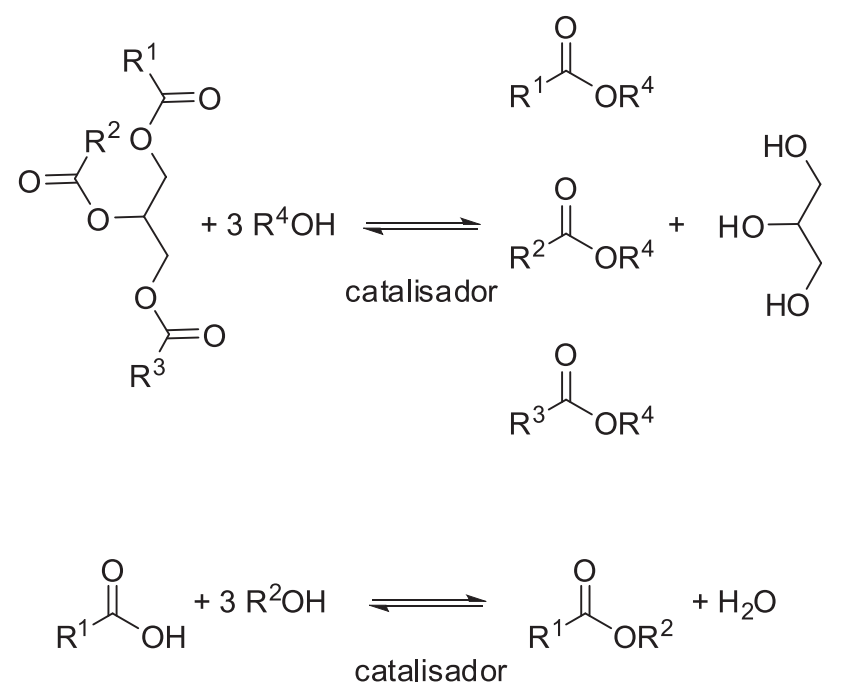

Esquema 1. Obtenção de ésteres graxos por transesterificação de triglicerídeos (superior) e por esterificação de ácidos graxos (inferior)

tem a tendência de formar cristais a baixas temperaturas..$^{8-10}$

A reação de transesterificação é uma substituição nucleofílica acílica $^{1,3,4}$ e pode ocorrer sob condições ácidas ou alcalinas. Vários óleos e gorduras podem ser usados como matérias-primas. ${ }^{1-6}$

Diversas atividades de laboratório para o ensino de graduação têm sido propostas na literatura incluindo a obtenção e caracterização do biodiesel. Óleos de soja, ${ }^{11-14}$ girassol, ${ }^{11}$ milho, ${ }^{11,15}$ amendoim,,${ }^{16}$ oliva,,${ }^{17}$ pinhão-manso, ${ }^{18}$ canola $^{19}$ e residual ${ }^{13,15,20}$ têm sido empregados. Álcool metílico tem sido o mais usado, ${ }^{11-13,16-21}$ embora alcoóis etílico ${ }^{14} \mathrm{e}$ $n$-propílico ${ }^{15}$ tenham também sido sugeridos. Catálises alcalina com $\mathrm{NaOH},{ }^{11,14,16,21} \mathrm{KOH}^{12,13,19,20}$ e $\mathrm{K}_{2} \mathrm{CO}_{3}{ }^{18}$ e ácida com $\mathrm{H}_{2} \mathrm{SO}_{4}{ }^{15}$ foram 
reportadas. Além disso, a combinação dos dois processos usando $\mathrm{H}_{2} \mathrm{SO}_{4}$ seguido de $\mathrm{CH}_{3} \mathrm{ONa}$ também foi descrita. ${ }^{17}$ Além do aquecimento convencional, a técnica de micro-ondas foi ainda sugerida. ${ }^{19}$ Nestas propostas, o biodiesel foi caracterizado por RMN-1 $\mathrm{H},{ }^{11-13,15,19}$ IV,${ }^{11,18}$ calor de combustão e ponto de névoa, ${ }^{16}$ glicerina livre e total, ${ }^{17}$ ponto de fulgor, ${ }^{20}$ densidade,,${ }^{12,20} \mathrm{e}$, ainda, cromatografia de camada delgada ${ }^{12,13}$ e viscosidade relativa. ${ }^{11,14,15,18}$ Entretanto, apesar desta larga aplicação da reação de obtenção de biodiesel em aulas experimentais da graduação, as avaliações da pureza e da composição jamais foram exploradas.

Neste trabalho, são apresentadas propostas de aulas experimentais em que os alunos obtêm biodiesel metílico a partir de diversas matérias-primas e também determinam a sua composição e pureza por cromatografia em fase gasosa. Além disso, a conversão dos triglicerídeos em ésteres graxos pode, ainda, ser facilmente confirmada por cromatografia de camada delgada, massa específica e viscosidade relativa.

A literatura é bastante rica e materiais bibliográficos são facilmente encontrados, facilitando ao professor a introdução do tópico e sua contextualização e auxiliando aos estudantes na compreensão do experimento e na elaboração de relatórios. São inúmeros os artigos, as revisões e as notas técnicas compreendendo temas como energia, ${ }^{22,23}$ óleos e gorduras, ${ }^{24-26}$ biodiesel, ${ }^{1-7,27-29}$ transesterificação, ${ }^{1-5,27-29}$ catálise, ${ }^{2,30}$ história, ${ }^{5,28}$ propriedades, métodos analíticos e especificações, , $, 8-10,31,32$ cromatografia ${ }^{33-38}$ e glicerol, ${ }^{39-41}$ apenas como alguns exemplos.

O procedimento experimental envolve um grande número de operações incluindo refluxo, evaporação à pressão reduzida, filtração, lavagem, titulação e cromatografia de camada delgada, entre outras.

No laboratório, os estudantes sintetizaram e caracterizaram biodieseis de óleos de palma, soja, canola, milho, arroz, uva, amendoim, girassol, pequi e oliva, além de sebo e banha. As transesterificações foram conduzidas em duas etapas consecutivas, usando $\mathrm{KOH}$ seguido de $\mathrm{H}_{2} \mathrm{SO}_{4}$ como catalisadores. Os procedimentos e resultados permitiram a discussão dos seguintes tópicos: óleos e gorduras, fontes, composição e propriedades; substituições nucleofílicas acílicas sob condições alcalinas e ácidas; cromatografia de camada delgada e sua aplicação no acompanhamento de reações; e cromatografia gasosa e seus métodos quantitativos (normalização de áreas e padronização interna).

\section{PARTE EXPERIMENTAL}

O procedimento experimental foi testado em cinco turmas de Análise e Síntese Orgânica do curso de Química (ULBRA, Canoas) e em atividades de extensão em diversas ocasiões. As atividades foram realizadas individualmente ou em grupos de dois ou três estudantes em duas aulas práticas de $3 \mathrm{~h}$. Os dados foram tratados e discutidos em uma aula adicional. Cada grupo utilizou uma matéria-prima diferente. $\mathrm{Na} 1^{\mathrm{a}}$ aula, a transesterificação foi conduzida. $\mathrm{O}$ metanol e o triglicerídeo foram aquecidos a $65{ }^{\circ} \mathrm{C}$ na presença de $\mathrm{KOH}$ por $1 \mathrm{~h}$. Na sequência, uma solução de $\mathrm{H}_{2} \mathrm{SO}_{4}$ em metanol foi adicionada sobre a mistura reacional, a qual foi mantida no aquecimento por $1 \mathrm{~h}$ adicional, segundo o protocolo original de Samios e colaboradores. ${ }^{42}$ Durante o período de refluxo, os estudantes determinaram o índice de acidez dos triglicerídeos. Aqueles grupos que utilizaram óleos ainda estimaram sua massa específica. Na segunda aula, as demais atividades foram realizadas.

\section{RESULTADOS E DISCUSSÃO}

O procedimento de transesterificação é simples e não ofereceu dificuldade aos estudantes. A metodologia associa a rapidez da catálise alcalina com a alta conversão da catálise ácida. A inversão de $\mathrm{pH}$ permite a esterificação dos sabões eventualmente formados na primeira etapa e torna a separação do biodiesel e da glicerina mais fácil.

A análise por cromatografia de camada delgada (CCD) evidencia a conversão da matéria-prima no produto desejado. Soluções de biodiesel e triglicerídeo foram separadamente preparadas e aplicadas lado a lado na cromatoplaca de silicagel, a qual foi eluída com uma mistura de hexano, éter etílico e HOAc (80:19:1) e revelada sob vapor de $\mathrm{I}_{2} \cdot{ }^{43}$ Nestas condições, biodiesel e triglicerídeo apresentaram fatores de retenção $\left(R_{F}\right)$ de 0,8 e 0,7 , respectivamente. Em todos os casos não se observou a presença da matéria-prima no produto, o que indica alta conversão e pureza elevada.

Valores típicos dos resultados experimentais obtidos pelos estudantes são apresentados na Tabela 1. As propriedades foram determinadas em triplicata e expressas com intervalos de confiança de $95 \%$.

O índice de acidez (IA) é a quantidade de base, expressa como miligramas de $\mathrm{KOH}$ por grama de amostra, necessária para titular o triglicerídeo e está relacionado com a presença de ácidos graxos livres no óleo ou na gordura. ${ }^{44} \mathrm{O}$ índice de acidez deve ser suficientemente baixo para que não haja o consumo do catalisador na etapa alcalina da transesterificação promovendo a formação de sabão. ${ }^{2}$ Em alguns casos, os estudantes tiveram dificuldade na visualização do ponto final da titulação, já que a amostra apresenta cor e a neutralização ocorre em sistema bifásico.

As massas específicas do óleo e do biodiesel foram estimadas em balões volumétricos de $10 \mathrm{~mL}$. Os valores encontrados são semelhantes aos relatados na literatura, ${ }^{32} 915$ e $870 \mathrm{~kg} \mathrm{~m}^{-3}$, respectivamente. A

Tabela 1. Resultados experimentais: índice de acidez da matéria-prima ( $\left.\mathrm{IA}_{\mathrm{TG}}\right)$, massa específica da matéria-prima $\left(\rho_{\mathrm{TG}}\right)$ e do biodiesel $\left(\rho_{\mathrm{BD}}\right)$, viscosidade relativa $\left(\eta_{\mathrm{REL}}\right)$, pureza do biodiesel $(\mathrm{P})$ e rendimento da reação $(\mathrm{R})$

\begin{tabular}{|c|c|c|c|c|c|c|}
\hline Materia-prima & $\mathrm{IA}_{\mathrm{TG}} /\left(\mathrm{mg} \mathrm{KOH} \mathrm{g}^{-1}\right)$ & $\rho_{\mathrm{TG}} /\left(\mathrm{kg} \mathrm{m}^{-3}\right)$ & $\rho_{\mathrm{BD}} /\left(\mathrm{kg} \mathrm{m}^{-3}\right)$ & $\eta_{\text {REL }}$ & $\mathrm{P} /(\%)$ & $\mathrm{R} /(\%)$ \\
\hline uva & $0,20 \pm 0,01$ & $917 \pm 3$ & $882 \pm 2$ & $9,0 \pm 0,4$ & $96,3 \pm 0,5$ & 93,1 \\
\hline soja & $0,069 \pm 0,008$ & $915 \pm 1$ & $879 \pm 2$ & $9,0 \pm 0,6$ & $100,9 \pm 0,9$ & 94,4 \\
\hline arroz & $0,5 \pm 0,2$ & $917 \pm 1$ & $876 \pm 5$ & $9,4 \pm 0,4$ & $95,0 \pm 2,6$ & 90,5 \\
\hline canola & $0,08 \pm 0,02$ & $915 \pm 3$ & $877 \pm 2$ & $9,3 \pm 0,4$ & $94,2 \pm 2,9$ & 85,3 \\
\hline milho & $0,09 \pm 0,04$ & $917 \pm 3$ & $876 \pm 2$ & $8,9 \pm 0,4$ & $100,1 \pm 1,4$ & 89,4 \\
\hline girassol & $0,0896 \pm 0,0003$ & $918 \pm 8$ & $875 \pm 7$ & $7,1 \pm 0,3$ & $87,8 \pm 1,2$ & 86,7 \\
\hline amendoim & $0,08 \pm 0,01$ & $911 \pm 3$ & $865 \pm 7$ & $9,8 \pm 0,4$ & $96,9 \pm 2,8$ & 95,7 \\
\hline oliva & $0,05 \pm 0,02$ & $910 \pm 1$ & $874 \pm 4$ & $7 \pm 1$ & $96,3 \pm 1,6$ & 90,0 \\
\hline palma & $11,6 \pm 0,2$ & - & $871 \pm 5$ & - & $95,2 \pm 4,6$ & 91,3 \\
\hline pequi & $2,2 \pm 0,1$ & $911 \pm 1$ & $971 \pm 1$ & - & $86,5 \pm 3,0$ & 90,9 \\
\hline banha & $2,1 \pm 0,1$ & - & $871 \pm 3$ & - & $97,0 \pm 3,1$ & 95,4 \\
\hline sebo & $2,56 \pm 0,09$ & - & $867 \pm 1$ & - & $97,6 \pm 2,2$ & 96,3 \\
\hline
\end{tabular}

Resultados expressos em intervalos de confiança de $95 \%$. 
Tabela 2. Teor de ésteres graxos no biodiesel (\%)

\begin{tabular}{|c|c|c|c|c|c|c|c|c|c|}
\hline biodiesel & C14:0 & C16:0 & C16:1 & C17:0 & C18:0 & C18:1 & C18:2 & C18:3 & Outros \\
\hline uva & 0,0 & 7,1 & 0,1 & 0,0 & 3,4 & 20,9 & 66,0 & 0,6 & 2,0 \\
\hline soja & 0,1 & 10,5 & 0,1 & 0,0 & 2,0 & 24,9 & 53,4 & 4,6 & 4,6 \\
\hline arroz & 0,2 & 21,3 & 0,1 & 0,0 & 1,2 & 36,7 & 37,2 & 1,2 & 2,1 \\
\hline canola & 0,1 & 4,8 & 0,2 & 0,0 & 1,3 & 64,5 & 20,9 & 5,2 & 3,0 \\
\hline milho & 0,0 & 11,9 & 0,1 & 0,0 & 1,2 & 35,9 & 49,3 & 0,0 & 1,6 \\
\hline palma & 0,9 & 41,6 & 0,1 & 0,1 & 3,2 & 41,5 & 12,0 & 0,2 & 0,5 \\
\hline girassol & 0,1 & 6,6 & 0,1 & 0,0 & 2,9 & 33,8 & 55,0 & 0,2 & 1,4 \\
\hline amendoim & 0,1 & 8,3 & 0,0 & 0,0 & 0,5 & 62,4 & 21,9 & 0,7 & 6,1 \\
\hline oliva & 0,4 & 18,4 & 4,8 & 0,0 & 4,8 & 34,4 & 31,7 & 3,8 & 1,8 \\
\hline pequi & 0,1 & 37,1 & 1,4 & 0,0 & 0,7 & 57,8 & 1,7 & 0,2 & 1,0 \\
\hline banha & 1,3 & 22,6 & 3,5 & 0,5 & 9,2 & 41,8 & 17,4 & 0,5 & 3,2 \\
\hline sebo & 3,2 & 25,2 & 3,6 & 1,2 & 15,7 & 42,0 & 0,8 & 0,6 & 7,6 \\
\hline
\end{tabular}

viscosidade relativa $\left(\eta_{\text {REL }}\right)$ foi determinada como a razão dos tempos de escoamento do óleo e do biodiesel medidos em pipetas volumétricas de $5 \mathrm{~mL} \cdot{ }^{11,14,15} \mathrm{~A}$ viscosidade do óleo, como esperado, é cerca de 10 vezes superior à do biodiesel. Ambas as propriedades não se mostraram influenciadas pela natureza da matéria-prima.

A pureza (P) do biodiesel foi estimada como o teor de ésteres graxos por cromatografia gasosa com padronização interna e calibração por um ponto..$^{45}$ Os estudantes prepararam suas amostras, as quais foram encaminhadas para análise fora do período de aula. No encontro seguinte, em aula teórica, os cromatogramas foram devolvidos e os dados foram tratados e discutidos. Purezas superiores a $90 \%$ são normalmente observadas pelos estudantes. O rendimento (R) foi estimado como o produto da razão das massas de biodiesel e triglicerídeo com a pureza.

A composição do biodiesel foi determinada por normalização das áreas dos picos. Os picos foram identificados pelos tempos de retenção $\left(t_{R}\right)$ por comparação com aqueles obtidos para padrões comerciais. Resultados típicos são apresentados na Tabela 2.

Os ácidos graxos ocorrem como ésteres da glicerina nos triglicerídeos e a composição não deve sofrer alteração durante a transesterificação. Assim, o teor de cadeias insaturadas no biodiesel pode ser utilizado para discutir a diferença de estado físico entre os óleos e as gorduras. ${ }^{4,24,25}$ Sebo e banha, por exemplo, sólidos à temperatura ambiente, apresentam cadeias insaturadas até $63 \%$. Nos óleos, por outro lado, o teor destas cadeias sobe para a faixa de $75 \%$ no óleo de arroz e no azeite de oliva, chegando até $91 \%$ no óleo de canola. A composição foi também utilizada para a discussão do efeito do teor de cadeias insaturadas no ponto de entupimento de filtro a frio e estabilidade oxidativa.

O protocolo original de transesterificação foi recentemente otimizado pelos seus autores ${ }^{46} \mathrm{e}$ estendido à rota etílica ${ }^{47}$ As modificações, entretanto, não foram ainda testadas na disciplina.

O material suplementar apresenta o procedimento experimental detalhado e é acompanhado por um estudo dirigido no qual os seguintes temas foram introduzidos: o biodiesel, os triglicerídeos, a reação de transesterificação e técnicas cromatográficas. Ao final de cada experimento, são apresentadas questões para discussão de resultados. Há recomendação de literatura de fáceis acesso e compreensão.

\section{CONCLUSÕES}

O conjunto de atividades que compõe o experimento pode ser desenvolvido em duas aulas de laboratório, ficando parte das discussões e a conclusão do projeto para uma aula teórica adicional. Os estudantes prepararam e caracterizaram biodieseis metílicos a partir de doze fontes de triglicerídeos. As diferenças de propriedades entre a matéria-prima e os ésteres graxos foram discutidas e utilizadas como evidências da transesterificação. Cromatografia gasosa foi utilizada na determinação da pureza e da composição dos biodieseis e, por consequência, também de suas matérias-primas. Os materiais utilizados, exceto na análise por cromatografia gasosa, são baratos e facilmente adquiridos. Aspectos como a natureza dos óleos e gorduras, a reação de transesterificação e a aplicação da cromatografia gasosa como técnica de análise quantitativa foram discutidos. Além disso, o experimento também oportuniza o debate sobre temas atuais como energia, sustentabilidade, meio ambiente e produção agrícola, entre outros.

\section{MATERIAL SUPLEMENTAR}

Os procedimentos experimentais e observações ao professor podem ser obtidos como material suplementar em http//quimicanova. sbq.org.br, em arquivo pdf e com acesso livre.

\section{AGRADECIMENTO}

À FAPERGS pela bolsa de iniciação tecnológica de D. M. de Oliveira e à PROPESQ/ULBRA pela bolsa de IC de V. O. B. dos Santos.

\section{REFERÊNCIAS}

1. Schuchardt, U.; Sercheli, R.; Vargas, R. M.; J. Braz. Chem. Soc. 1998, 9, 199.

2. Pinto, A. C.; Guarieiro, L. L. N.; Rezende, M. J. C.; Ribeiro, N. M.; Torres, E. A.; Lopes, W. A.; Pereira, P. A. P.; de Andrade, J. B.; J. Braz. Chem. Soc. 2005, 16, 1313.

3. Suarez, P. A. Z.; Meneghetti, S. M. P.; Meneghetti, M. R.; Wolf, C. R.; Quim. Nova 2007, 30, 667 .

4. Marques, M. V.; Naciuk, F. F.; da Silva, C. F. G.; Fontoura, L. A. M.; Analytica 2008, 33, 72.

5. Oliveira, F. C. C.; Suarez, P. A. Z.; dos Santos, W. L. P.; Química Nova na Escola 2008, 28, 3.

6. Demirbas, A.; Energy Conv. Manag. 2008, 49, 2106.

7. King, A. G.; Wright, M. W.; J. Chem. Educ. 2007, 84, 202.

8. Knothe, G.; Fuel Process. Technol. 2005, 86, 1059. 
9. Knothe, G.; Fuel Process. Technol. 2007, 88, 669.

10. Knothe, G.; Energy Fuels 2008, 22, 1358.

11. Clarke, N. R.; Casey, J. P.; Brown, E. D.; Oneyma, E.; Donaghy, K. J.; J. Chem. Educ. 2006, 83, 257.

12. Géris, R.; dos Santos, N. A. C.; Amaral, B. A.; Maia, I. S.; Castro, V. D.; Carvalho, J. R. M.; Quim. Nova 2007, 30, 1369.

13. Rinaldi, R.; Garcia, C.; Marciniuk, L. L.; Rossi, A. V.; Schuchardt, U.; Quim. Nova 2007, 30, 1374.

14. Santos, A. P. B.; Pinto, A. C.; Química Nova na Escola 2009, 31, 58.

15. Bladt, D.; Murray, S.; Gitch, B.; Trout, H.; Liberko, C.; J. Chem. Educ. 2011, $88,201$.

16. Akers, S. M.; Conkle, J. L.; Thomas, S. N.; Rider, K. B.; J. Chem. Educ. 2006, 83, 260.

17. Bucholtz, E. C.; J. Chem. Educ. 2007, 84, 296.

18. Behnia, M. S.; Emerson, D. W.; Steinberg, S. M.; Alwis, R. M.; Dueñas, J. A.; Serafino, J. O.; J. Chem. Educ. 2011, 88, 1290.

19. Miller, T. A.; Leadbeater, N. E.; Chem. Educator 2009, 14, 98.

20. Hoffman, A. R.; Britton, S. L.; Cadwell, K. D.; Waltz, K. A.; J. Chem. Educ. 2011, 88, 197.

21. Meyer, S. A.; Morgenstern, M. A.; Chem. Educator 2005, 10, 130.

22. Goldenberg, J.; Quim. Nova 2009, 32, 582.

23. Vichi, F. M.; Mansor, M. T. C.; Quim. Nova 2009, 32, 757.

24. Vianni, R.; Braz-Filho, R.; Quim. Nova 1996, 19, 400.

25. Reda, S. Y.; Carneiro, P. I. B.; Analytica 2007, 27, 60.

26. Merçon, F.; Química Nova na Escola. 2010, 32, 78.

27. Costa Neto, P. R.; Rossi, L. F. S.; Zagonel, G. F.; Ramos, L. P.; Quim. Nova 2000, 23, 531 .

28. Suarez, P. A. Z.; Meneghetti, S. M. P.; Quim. Nova 2007, 30, 2068.

29. Suarez, P. A. Z.; Santos, A. L. F.; Rodrigues, J. P.; Alves, M. B.; Quim. Nova 2009, 32, 768 .

30. Cordeiro, C. S.; da Silva, F. R.; Wypych, F.; Ramos, L. P.; Quim. Nova 2011, 34, 477 .
31. Lôbo, I. P.; Ferreira, S. L. C.; da Cruz, R. S.; Quim. Nova 2009, 32, 1596.

32. Canakci, M.; Sanli, H.; J. Ind. Microbiol. Biotechnol. 2008, 35, 431.

33. Faria, R. C. M.; Rezende, M. J. C.; Rezende, C. M.; Pinto, A. C.; Quim. Nova 2007, 30, 1900.

34. Urioste, D.; Castro, M. B. A.; Biaggio, F. C.; de Castro, H. F.; Quim. Nova 2008, 31, 407

35. Marques, M. V.; Naciuk, F. F.; Mello, A. M. S.; Seibel, N. M.; Fontoura, L. A. M.; Quim. Nova 2010, 33, 978.

36. Visentainer, J. V.; Quim. Nova 2012, 35, 274.

37. Carvalho, M. S.; Mendonça, M. A.; Pinho, D. M. M.; Resck, I. S.; Suarez, P. A. Z.; J. Braz. Chem. Soc. 2012, 23, 763.

38. Froehner, S.; Leithold, J.; Lima Júnior, L. F.; Quim. Nova 2007, 30, 2016.

39. de Arruda, P. V.; Rodrigues, R. C. L. B.; Felipe, M. G. A.; Analytica 2007, 26, 56

40. Mota, C. J. A.; da Silva, C. X. A.; Gonçalves, V. L. L.; Quim. Nova 2007, 30, 639 .

41. Beatriz, A.; Araújo, Y. J. K.; de Lima, D. P.; Quim. Nova, 2011, 34, 306.

42. Samios, D.; Pedrotti, F.; Nicolau, A.; Reiznautt, Q. B.; Martini, D. D.; Dalcin, F. M.; Fuel Process. Technol. 2009, 90, 599.

43. Ferrari, R. A.; Oliveira, V. S.; Scabio, A.; Quim. Nova 2005, $28,19$.

44. Tubino, M.; Aricetti, J. A.; J. Braz. Chem. Soc. 2011, 22, 1073.

45. EN14103:2003; Fatty acid methyl esters (FAME) - Determination of ester and linolenic acid methyl ester contents, European Committee for Standardization: Brussels, 2003.

46. Guzatto, R.; de Martini, T. L.; Samios, D.; Fuel Process. Technol. 2011, 92, 2083.

47. Guzatto, R.; Defferrari, D.; Reiznautt, Q. B.; Cadore, I. R.; Samios, D.; Fuel 2012, 92, 197. 


\title{
OBTENÇÃO DE BIODIESEL POR TRANSESTERIFICAÇÃO EM DOIS ESTÁGIOS E SUA CARACTERIZAÇÃO POR CROMATOGRAFIA GASOSA: ÓLEOS E GORDURAS EM LABORATÓRIO DE QUÍMICA ORGÂNICA
}

\author{
Diogo Müller de Oliveira, Diego Paulo Ongaratto, Luiz Antonio Mazzini Fontoura*, Fabrício Fredo Naciuk, Vinícius \\ Oliveira Batista dos Santos, Jéssica Danieli Kunz e Marcelo Volpatto Marques \\ Departamento de Engenharia de Processos, Fundação de Ciência e Tecnologia, Rua Washington Luiz, 675, 90010-460 Porto Alegre \\ - RS / Curso de Química, Universidade Luterana do Brasil, Rua Farroupilha, 8001, 92425-900 Canoas - RS, Brasil \\ Alexander Ossanes de Souza e Claudio Martin Pereira de Pereira \\ Centro de Ciências Químicas, Farmacêuticas e de Alimentos, Universidade Federal de Pelotas, Campus Universitário, Capão do \\ Leão, s/n, 96010-900 Pelotas - RS, Brasil
}

Dimitrios Samios

Departamento de Físico-Química, Instituto de Química, Universidade Federal do Rio Grande do Sul, Av. Bento Gonçalves, 9500 , 91501-970 Porto Alegre - RS, Brasil

\section{PROCEDIMENTO EXPERIMENTAL E ESTUDO DIRIGIDO}

Neste projeto, você terá a oportunidade de obter biodiesel e caracterizá-lo. Siga as normas de segurança e a orientação do seu professor. Trabalhe na capela. Utilize avental e óculos de segurança.

Atenção: metanol, éter etílico e heptano são líquidos inflamáveis e tóxicos. Ácido sulfúrico, ácido acético glacial, hidróxido de sódio, hidróxido de potássio e iodo são corrosivos.

Junto com o procedimento experimental, você está recebendo um estudo dirigido. Busque a literatura sugerida e discuta as questões propostas com seus colegas. Há informações atualizadas no site da Agência Nacional de Petróleo, Gás Natural e Biocombustíveis que podem ajudá-lo. Bom trabalho!

http://www.anp.gov.br/?pg=61929\&m=\&t1=\&t2=\&t3=\&t4=\&ar=\& ps $=\&$ cachebust $=1350161456419$

\section{Biodiesel}

\section{Você sabia?}

Biodiesel é um combustível de origem renovável composto por uma mistura de ésteres graxos, utilizado como substituinte parcial ou total ao diesel em motores com ignição por compressão. No Brasil, sua adição ao diesel fóssil tornou-se obrigatória em 2008. Hoje, é usado na proporção de $5 \%$ no combustível comercializado no país. O biodiesel apresenta diversas características vantajosas quando comparado com o diesel. Do ponto de vista ambiental, é livre de compostos de enxofre e aromáticos, tem uma menor contribuição no efeito estufa, é biodegradável e obtido a partir de fontes renováveis. Com relação aos aspectos econômicos, pode diminuir a dependência dos países importadores de petróleo daqueles que o produzem e estimular a produção agrícola e o desenvolvimento rural. No que diz respeito ao desempenho, o biodiesel apresenta maiores ponto de fulgor, número de cetano e poder lubrificante. Por fim, pode ser usado em motores com ignição por compressão sem que haja a necessidade de modificações mecânicas. Evidentemente, há algumas desvantagens. O poder calorífico, isto é, a quantidade de energia gerada na queima por unidade de massa do combustível, é menor do que no diesel. Além disso, degrada-se por oxidação com facilidade e tem a tendência de formar cristais a baixas temperaturas.

\section{Saiba mais!}

1. O que são biocombustíveis?

2. Quais são os principais biocombustíveis?
3. Qual é a posição do Brasil entre os países produtores de biodiesel?

Onde encontrar:

Suarez, P. A. Z.; Meneghetti, S. M. P.; Meneghetti, M. R.; Wolf, C. R.; Quim. Nova 2007, 30, 667 .

Marques, M. V.; Naciuk, F. F.; da Silva, C. F. G.; Fontoura, L. A. M.; Analytica 2008, 33, 72 .

\section{Triglicerídeos}

Você sabia?

O biodiesel é obtido normalmente a partir de triglicerídeos, os quais são os principais constituintes de óleos e gorduras. Estruturalmente, são ésteres do glicerol com ácidos graxos.

Saiba mais!

1. O que são ácidos graxos?

2. Quais são os principais ácidos graxos encontrados esterificados com a glicerina nos óleos e gorduras?

3. O que diferencia óleos e gorduras?

4. Qual a relação entre a insaturação das cadeias e o estado físico dos triglicerídeos?

5. Quais são as principais fontes de óleos e gorduras?

6. No Brasil, quais são as principis fontes de triglicerídeos utilizadas para a obtenção de biodiesel?

Onde encontrar?

Vianni, R.; Braz-Filho, R.; Quim. Nova 1996, 19, 400.

Reda, S. Y.; Carneiro, P. I. B.; Analytica 2007, 27, 60.

Merçon, F.; Química Nova na Escola 2010, 32, 78.

\section{Determinação do índice de acidez (IA) do triglicerídeo}

1. Em um erlenmeyer de $250 \mathrm{~mL}$, introduza $150 \mathrm{~mL}$ de álcool etílico e 2 gotas de solução de fenolftaleína.

2. Titule o álcool com $\mathrm{NaOH}$ aq. $0,1 \mathrm{~mol} \mathrm{~L}^{-1}$ até o aparecimento da primeira coloração rosa permanente por $30 \mathrm{~s}$.

3. Em um erlenmeyer de $250 \mathrm{~mL}$, pese ca $50 \mathrm{~g}$ do triglicerídeo escolhido. Utilize a tabela abaixo para anotar os seus dados.

4. A seguir, introduza $50 \mathrm{~mL}$ do álcool etílico neutralizado no erlenmeyer.

5. Adicione $1 \mathrm{~mL}$ de solução de fenolftaleína.

6. Com agitação magnética, aqueça a mistura a aproximadamente $60{ }^{\circ} \mathrm{C}$.

7. Titule com $\mathrm{NaOH} 0,1 \mathrm{~N}$ até o aparecimento de uma coloração rosa permanente por, ao menos, $30 \mathrm{~s}$. Anote o volume de titulante consumido. Faça a análise em triplicata. 
Para discutir

1. Complete a tabela com a massa em g de amostra e o volume em $\mathrm{mL}$ de solução do titulante consumido.

2. Em cada titulação, qual foi o número de móis (n) de $\mathrm{NaOH}$ consumido?

3. Se no lugar de $\mathrm{NaOH}$, você tivesse utilizado $\mathrm{KOH}$, qual massa de $\mathrm{KOH}$ em mg $\left(\mathrm{M}_{\mathrm{KOH}}\right)$ teria sido consumida?

4. O índice de acidez (IA), por definição é a massa de $\mathrm{KOH}$ em mg consumida para neutralizar $1 \mathrm{~g}$ de amostra. Determine o IA de cada replicata e expresse o resultado como a média das três titulações.

\begin{tabular}{|c|l|l|l|}
\hline titulação & 1 & 2 & 3 \\
\hline $\mathrm{m}(\mathrm{g})$ & & & \\
\hline $\mathrm{V}(\mathrm{mL})$ & & & \\
\hline $\mathrm{n}(\mathrm{mol})$ & & & \\
\hline $\mathrm{M}_{\mathrm{KOH}}(\mathrm{mg})$ & & & \\
\hline IA (mg KOH por g de amostra) & & & \\
\hline
\end{tabular}

5. Qual é a origem da acidez de sua amostra?

\section{Onde encontrar?}

Lôbo, I. P.; Ferreira, S. L. C.; da Cruz, R. S.; Quim. Nova 2009, 32, 1596.

Marques, M. V.; Naciuk, F. F.; da Silva, C. F. G.; Fontoura, L. A. M.; Analytica 2008, 33, 72.

Tubino, M.; Aricetti, J. A.; J. Braz. Chem. Soc. 2011, 22, 1073.

\section{Determinação da massa específica do triglicerídeo}

1. Tare um balão volumétrico de $10 \mathrm{~mL}$ e complete o volume até a marca com o triglicerídeo. Pese e calcule a massa específica ( $\rho)$. Faça a análise em triplicata. Anote os seus dados na tabela abaixo.

\begin{tabular}{|l|l|l|l|l|}
\hline Óleo vegetal & $\mathrm{M}_{1}(\mathrm{~g})$ & $\mathrm{M}_{2}(\mathrm{~g})$ & $\mathrm{M}_{3}(\mathrm{~g})$ & $\rho$ média $\left(\mathrm{g} \mathrm{mL}^{-1}\right)$ \\
\hline & & & & \\
\hline
\end{tabular}

\section{A reação de transesterificação}

\section{Você sabia?}

Triglicerídeos são ésteres e, como tais, reagem com nucleófilos por mecanismos de substituição nucleofílica acílica. A reação de transesterificação é um exemplo. O éster reage com um álcool gerando um novo éster e um novo álcool. O Esquema $1 \mathrm{~S}$ apresenta a obtenção de biodiesel por transesterificação de um triglicerídeo.

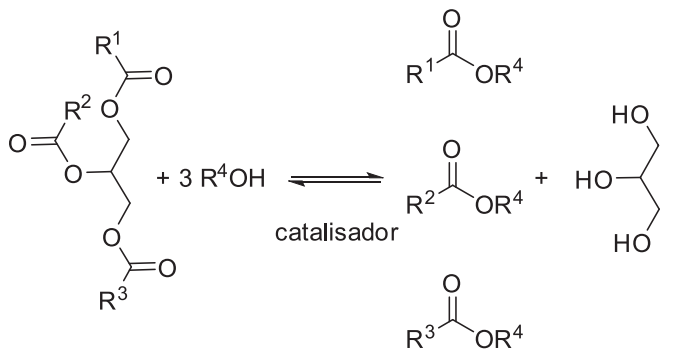

Esquema 1S. Obtenção de ésteres graxos por transesterificação de triglicerídeos

Ésteres são pouco reativos e a transesterificação, na ausência de um catalisador, é muito lenta. Bases como hidróxidos ou metóxidos de sódio ou potássio, ou, ainda, ácidos como o sulfúrico e o fosfórico podem ser utilizados como catalisadores.

Saiba mais!

1. Por que os ésteres são pouco reativos?
2. Apresente e discuta brevemente o mecanismo de uma transesterificação sob catálise alcalina.

3. Apresente e discuta brevemente o mecanismo de uma transesterificação sob catálise ácida.

4. Para que a transesterificação seja executada com catálise alcalina, é necessário que o índice de acidez seja baixo. Por quê?

5. Que vantagens e desvantagens a literatura apresenta para a obtenção de biodiesel por catálise alcalina ou ácida?

6. Que aplicações a literatura apresenta para a glicerina?

\section{Onde encontrar:}

Suarez, P. A. Z.; Meneghetti, S. M. P.; Meneghetti, M. R.; Wolf, C. R.; Quim. Nova 2007, 30, 667.

Marques, M. V.; Naciuk, F. F.; da Silva, C. F. G.; Fontoura, L. A. M.; Analytica 2008, 33, 72 .

Schuchardt, U.; Sercheli, R.; Vargas, R. M.; J. Braz. Chem. Soc. 1998, 9, 199. de Arruda, P. V.; Rodrigies, R. C. L. B.; Felipe, M. G. A.; Analytica 2007, $26,56$.

Mota, C. J. A.; da Silva, C. X. A.; Gonçalves, V. L. L.; Quim. Nova 2007, 30,639 .

Beatriz, A.; Araújo, Y. J. K.; de Lima, D. P.; Quim. Nova 2011, 34, 306.

\section{Obtenção de biodiesel por transesterificação de um} triglicerídeo

1. Realize a reação em capela.

2. Prepare um banho-maria em uma chapa de aquecimento provida de agitação magnética a $65-70{ }^{\circ} \mathrm{C}$. Assegure-se que a temperatura esteja estável neste intervalo.

3. Pese $c a 0,5 \mathrm{~g}$ de $\mathrm{KOH}$ em um béquer de $50 \mathrm{~mL}$. Anote os seus dados na tabela abaixo.

4. Sobre o sólido, adicione $20 \mathrm{~mL}$ de álcool metílico. Agite até a dissolução completa do sólido.

5. Pese ca $50 \mathrm{~mL}$ de óleo e transfira para um balão de fundo redondo de $125 \mathrm{~mL}$.

6. Adicione a solução alcoólica ao óleo.

7. Conecte um condensador de refluxo ao balão.

8. Aqueça a mistura no banho-maria, agitando-a vigorosamente por um período de $1 \mathrm{~h}$

9. Passado esse tempo, adicione ao balão, pela parte superior do condensador, uma mistura de $30 \mathrm{~mL}$ de álcool metílico e 0,75 $\mathrm{mL}$ de $\mathrm{H}_{2} \mathrm{SO}_{4}$.

10. Prossiga a reação por mais $1 \mathrm{~h}$.

11. Ao final deste tempo, filtre a mistura à pressão reduzida.

12. Transfira o filtrado para um balão de $125 \mathrm{~mL}$. Leve a mistura ao rotavapor para a eliminação do excesso de álcool.

13. Transfira a mistura para um funil de separação de $125 \mathrm{~mL}$.

14. Aguarde 15 min pela separação completa das fases. Separe e pese a fase inferior, a glicerina.

15. Introduza $25 \mathrm{~mL}$ de água deionizada a $70{ }^{\circ} \mathrm{C}$. Agite moderada e cuidadosamente a mistura. Não esqueça de aliviar a pressão interna abrindo ligeiramente a torneira durante o procedimento de lavagem.

16. Mantenha a mistura em repouso por $15 \mathrm{~min}$. Separe a fase inferior.

17. Repita as etapas 15 e 16.

18. Transfira o biodiesel para um balão de fundo redondo e leve novamente ao rotavapor para eliminação da umidade.

19. Pese o biodiesel obtido.

\begin{tabular}{|c|l|}
\hline triglicerídeo & \\
\hline$M_{\mathrm{KOH}}(\mathrm{g})$ & \\
\hline $\mathrm{M}_{\text {oleo }}(\mathrm{g})$ & \\
\hline $\mathrm{M}_{\text {glicerina }}(\mathrm{g})$ & \\
\hline $\mathrm{M}_{\text {biodiesel }}(\mathrm{g})$ & \\
\hline
\end{tabular}


Para discutir

1. O álcool e o triglicerídeo são imiscíveis. Da mesma forma, os produtos obtidos também não se misturam. Discuta.

2. O que é o sólido separado por filtração?

3. O álcool utilizado em excesso é recuperado no rotavapor. Por que o álcool é usado em excesso?

4. Qual é a aparência dos produtos obtidos?

5. Por que o biodiesel deve ser lavado?

6. Qual foi a relação percentual em massa entre o biodiesel e o triglicerídeo?

\section{Determinação da massa específica do Biodiesel}

1. Tare um balão volumétrico de $10 \mathrm{~mL}$ e complete o volume até a marca com o seu biodiesel. Pese e calcule a massa específica ( $\rho)$. Faça a análise em triplicata. Anote os seus dados na tabela abaixo.

\begin{tabular}{|l|l|l|l|l|}
\hline Biodiesel & $\mathrm{M}_{1}(\mathrm{~g})$ & $\mathrm{M}_{2}(\mathrm{~g})$ & $\mathrm{M}_{3}(\mathrm{~g})$ & $\rho$ média $\left(\mathrm{g} \mathrm{mL}^{-1}\right)$ \\
\hline & & & & \\
\hline
\end{tabular}

Para discutir

1. Que fatores afetam a massa específica do biodiesel?

2. Compare as massas específicas do biodiesel e do triglicerídeo utilizado. A que você atribui a diferença?

3. Qual é a faixa de massa específica aceita para o biodiesel? O valor que você encontrou está dentro desta faixa? Baseado nestas informações, você pode afirmar que teve sucesso em sua reação?

\section{Onde encontrar:}

Lôbo, I. P.; Ferreira, S. L. C.; da Cruz, R. S.; Quim. Nova 2009, 32, 1596.

Marques, M. V.; Naciuk, F. F.; da Silva, C. F. G.; Fontoura, L. A. M.; Analytica 2008, 33, 72.

\section{Determinação da viscosidade relativa do Biodiesel}

1. Com uma caneta marcador, trace uma linha horizontal próximo à ponta inferior de uma pipeta volumétrica de $5 \mathrm{~mL}$.

2. Com o auxílio de uma pera de sucção, introduza o óleo na pipeta até acima da marca de volume.

3. Prenda a pipeta na posição vertical a um suporte para buretas.

4. Desconecte a pera da pipeta de modo a permitir o escoamento do líquido.

5. Acione o cronômetro no momento em que o líquido atravessar a marca superior da pipeta. Desligue o cronômetro quando o menisco atingir a marca inferior

6. Anote o tempo na tabela abaixo.

7. Execute a medida em triplicata.

8. Lave a pipeta com pequena quantidade de heptano.

9. Aguarde a pipeta secar.

10. Repita o procedimento com uma amostra do seu biodiesel.

11. Expresse a viscosidade relativa $\left(\eta_{\mathrm{REL}}\right)$ como a razão entre os tempos médios de escoamento do óleo e do biodiesel.

\begin{tabular}{|c|c|c|}
\hline $\mathrm{t}(\mathrm{s})$ & óleo & biodiesel \\
\hline $\mathrm{t}_{1}$ & & \\
\hline $\mathrm{t}_{2}$ & & \\
\hline $\mathrm{t}_{3}$ & & \\
\hline $\mathrm{t}_{\text {médio }}$ & & \\
\hline$\eta_{\text {REL }}$ & & \\
\hline
\end{tabular}

Para discutir

1. Que fatores afetam a viscosidade do biodiesel?

2. Compare os tempos de escoamento do biodiesel e do triglicerídeo utilizado. A que você atribui a diferença?
Onde encontrar:

Lôbo, I. P.; Ferreira, S. L. C.; da Cruz, R. S.; Quim. Nova 2009, 32, 1596.

Marques, M. V.; Naciuk, F. F.; da Silva, C. F. G.; Fontoura, L. A. M.; Analytica 2008, 33, 72 .

\section{A cromatografia}

Você sabia?

Cromatografia corresponde a uma família de técnicas que tem por objetivo primário a separação dos componentes de uma mistura. $\mathrm{O}$ processo de separação baseia-se na disputa de duas fases chamadas de estacionária e móvel por cada um dos componentes da mistura. A cromatografia pode ser usada para análise quali e quantitativa.

Saiba mais!

1. O que é a cromatografia da camada delgada?

2. Qual o cuidado que se deve ter ao aplicar a amostra sobre a cromatoplaca?

3. Como se determina o fator de retenção?

4. Quais são as formas mais comuns de revelação da placa?

5. Quais são os principais componentes de um cromatógrafo gasoso?

6. Quais são as principais características das colunas capilares?

7. Quais são os principais detectores em cromatografia gasosa?

8. Quais são os gases de arraste normalmente utilizados?

Onde encontrar:

Degani, A. L. G.; Cass, Q. B. Vieira, P. C.; Química Nova na Escola 1998, $\mathrm{n}^{\circ} 7,21$.

VI. Cromatografia de camada delgada do biodiesel (CCD)

1. Prepare o eluente misturando $8 \mathrm{~mL}$ de heptano, $2 \mathrm{~mL}$ de éter etílico e 1 gota de ácido acético glacial.

2. Prepare a cuba para a CCD.

3. Em um tubo de ensaio, adicione $1 \mathrm{~mL}$ de heptano seguido por 1 gota do biodiesel.

4. Utilize o mesmo procedimento para preparar uma amostra da matéria-prima utilizada.

5. Aplique, com o auxílio de capilares, lado a lado em uma cromatoplaca, as amostras de triglicerídeo e de biodiesel.

6. Elua a placa.

7. Revele em câmara de iodo.

8. Represente abaixo a sua cromatoplaca após a revelação.

9. Determine os fatores de retenção $\left(R_{F}\right)$ para matéria-prima e produto.

Para discutir

1. Há triglicerídeo residual no seu biodiesel?

2. O que ocorreria com os fatores de retenção do triglicerídeo e do biodiesel se a proporção de éter etílico fosse aumentada no eluente?

Onde encontrar:

Ferrari, R. A.; Oliveira, V. S.; Scabio, A.; Quim. Nova 2005, 28, 19.

Froehner, S.; Leithold, J.; Lima Júnior, L. F.; Quim. Nova 2007, 30, 2016.

\section{Determinação da pureza do biodiesel}

Preparação da solução de heptadecanoato de metila ca $10 \mathrm{mg} \mathrm{mL}^{-1}$ (padrão interno)

1. Pese com exatidão, diretamente para um balão volumétrico de $25 \mathrm{~mL}$, uma massa de ca $250 \mathrm{mg}$ de heptadecanoato de metila.

2. A seguir, adicione heptano ao balão até a marca. Homogeneíze a mistura. 


\section{Preparação da amostra}

1. Pese com exatidão, diretamente para um frasco de $10 \mathrm{~mL}$, uma massa de ca $250 \mathrm{mg}$ de amostra.

2. A seguir, sobre a amostra, adicione $5 \mathrm{~mL}$ de solução padrão.

3. Homogeneíze.

4. Prepare a amostra em triplicata.

\section{Análise cromatográfica}

1. Obtenha com o seu professor as condições de análise cromatográfica.

2. Injete 3 vezes cada replicata.

\begin{tabular}{|l|l|}
\hline Cromatógrafo/Detector & \\
\hline Temperatura do injetor $\left({ }^{\circ} \mathrm{C}\right)$ & \\
\hline Coluna & \\
\hline Vazão $\left(\mathrm{mL} \mathrm{min}^{-1}\right)$ & \\
\hline Temperatura inicial $-\mathrm{T}_{\mathrm{i}}\left({ }^{\circ} \mathrm{C}\right)$ & \\
\hline Tempo na $\mathrm{T}_{\mathrm{i}}(\mathrm{min})$ & \\
\hline Taxa de aquecimento $\left({ }^{\circ} \mathrm{C} \min ^{-1}\right)$ & \\
\hline Temperatura final $-\mathrm{T}_{\mathrm{F}}\left({ }^{\circ} \mathrm{C}\right)$ & \\
\hline Tempo na $\mathrm{T}_{\mathrm{F}}(\min )$ & \\
\hline Gás de arraste & \\
\hline Temperatura do detector $\left({ }^{\circ} \mathrm{C}\right)$ & \\
\hline Split & \\
\hline Volume injetado $(\mu \mathrm{L})$ & \\
\hline
\end{tabular}

\section{Expressão do resultado}

1. Determine a pureza do biodiesel, expressa como teor de ésteres graxos metílicos, pela Equação 3,

Teor de ésteres graxos $(\%)=\frac{\sum A-A_{P I}}{A_{P I}} \times \frac{C_{P I} \times V_{P i}}{M} \times 100$

na qual, $A$ é a área total integrada entre os picos identificados como C14:0 e C24:1; $A_{P I}$ é a área do pico identificado como padrão interno; $C_{P I}$ é a concentração da solução de padrão interno em $\mathrm{mg} \mathrm{mL}^{-1} ; V_{P I}$ é o volume usado da solução de padrão interno no preparo da amostra em $\mathrm{mL}$; $M$ é a massa de amostra em $\mathrm{mg}$.

Utilize A e $\mathrm{A}_{\mathrm{PI}}$ como a média de três injeções.

2. Expresse o resultado com uma casa decimal.

\begin{tabular}{|c|c|c|c|}
\hline \multirow{2}{*}{$\begin{array}{l}\text { Replicata } \\
\mathrm{t}_{\mathrm{R}}(\min ) \mathrm{PI}\end{array}$} & 1 & 2 & 3 \\
\hline & & & \\
\hline $\mathrm{A}_{1^{a} \text { injeção }}$ & & & \\
\hline$A_{2^{n} \text { injeç̃o }}$ & & & \\
\hline$A_{3^{n} \text { injeção }}$ & & & \\
\hline A & & & \\
\hline $\mathrm{A}_{\mathrm{PI} 1^{a} \text { injeção }}$ & & & \\
\hline $\mathrm{A}_{\mathrm{PI} 2^{\mathrm{a}} \text { injeção }}$ & & & \\
\hline $\mathrm{A}_{\mathrm{PI3^{a }} \text { injecãa }}$ & & & \\
\hline $\mathrm{A}_{\mathrm{PI}}$ & & & \\
\hline $\mathrm{C}_{\mathrm{PI}}\left(\mathrm{mg} \mathrm{mL}^{-1}\right)$ & & & \\
\hline $\mathrm{V}_{\mathrm{PI}}(\mathrm{mL})$ & & & \\
\hline $\mathrm{M}(\mathrm{mg})$ & & & \\
\hline Teor de ésteres graxos (\%) & & & \\
\hline
\end{tabular}

\section{Para discutir}

1. Quais são os principais contaminantes do biodiesel?

2. Que características deve apresentar um padrão interno?

3. Por que o heptadecanoato de metila é usado como padrão interno na determinação da pureza do biodiesel?

4. Qual é a pureza mínima aceitável para o biodiesel?
5. O que ocorreria com os tempos de retenção dos diversos ésteres graxos se a temperatura de forno fosse aumentada? E se fosse aumentada a vazão do gás de arraste?

Onde encontrar:

Marques, M. V.; Naciuk, F. F.; Mello, A. M. S.; Seibel, N. M.; Fontoura, L. A. M.; Quim. Nova 2010, 33, 978.

Lôbo, I. P.; Ferreira, S. L. C.; da Cruz, R. S.; Quim. Nova 2009, 32, 1596.

Marques, M. V.; Naciuk, F. F.; da Silva, C. F. G.; Fontoura, L. A. M.; Analytica 2008, 33, 72.

\section{Determinação da composição do biodiesel}

1. Examine o cromatograma de sua amostra e identifique os principais picos pelos tempos de retenção $\left(t_{R}\right)$ dos padrões injetados previamente. Consulte seu professor.

2. Complete a tabela com os tempos de retenção $\left(t_{R}\right)$ e as áreas relativas de cada pico e calcule o teor de cada éster graxo pela Equação 4, na qual A é a área total menos a área do padrão interno e $\mathrm{A}_{\mathrm{i}} \mathrm{a}$ área relativa de cada pico.

$$
\text { Teor de ésteres graxos }(\%)=\frac{100 \times A_{i}}{A}
$$

\begin{tabular}{|l|c|c|c|c|c|c|}
\hline & $\begin{array}{c}\text { miristato } \\
(\mathrm{C} 14: 0)\end{array}$ & $\begin{array}{c}\text { palmitato } \\
(16: 0)\end{array}$ & $\begin{array}{c}\text { estearato } \\
(18: 0)\end{array}$ & $\begin{array}{c}\text { oleato } \\
(18: 1)\end{array}$ & $\begin{array}{c}\text { linoleato } \\
(18: 2)\end{array}$ & $\begin{array}{c}\text { linolenato } \\
(\mathrm{C} 18: 3)\end{array}$ \\
\hline $\mathrm{t}_{\mathrm{R}}(\mathrm{min})$ & & & & & & \\
\hline $\mathrm{A}_{\mathrm{i}}$ & & & & & & \\
\hline$\% \mathrm{RCOOMe}$ & & & & & & \\
\hline
\end{tabular}

\section{Para discutir}

1. Compare a composição do seu biodiesel com o de seus colegas.

2. Qual é o teor aproximado de ésteres graxos saturados no seu biodiesel?

3. Qual é o teor aproximado de ésteres graxos poliinsaturados no seu biodiesel?

4. O que é o ponto de entupimento de filtro a frio? De que forma a composição afeta esta propriedade?

5. O que é a estabilidade à oxidação? Que fatores afetam esta propriedade?

6. Qual é a relação entre a composição do biodiesel e sua estabilidade à oxidação?

Onde encontrar:

Lôbo, I. P.; Ferreira, S. L. C.; da Cruz, R. S.; Quim. Nova 2009, 32, 1596.

Marques, M. V.; Naciuk, F. F.; da Silva, C. F. G.; Fontoura, L. A. M.; Analytica 2008, 33, 72.

IX. Cálculo da massa molar média $\left(\mathrm{MMM}_{\mathrm{TG}}\right)$ do triglicerídeo

1. Os grupos acila $(\mathrm{R}-\mathrm{C}=\mathrm{O})$ encontrados nos triglicerídeos são derivados dos mais variados ácidos graxos e em diferentes proporções. Assim, na matéria-prima que você utilizou não há um único triglicerídeo, mas uma mistura deles, contendo quaisquer dos resíduos de ácidos graxos, em qualquer ordem e proporção. Não há, portanto, uma massa molar do triglicerídeo, mas uma massa molar média, que é obtida a partir do percentual de cada ácido graxo presente.

2. Você já calculou a composição do seu biodiesel (\% RCOOH). Transporte os valores para a tabela a seguir.

3. Calcule a massa molar dos resíduos de glicerina $\left(\mathrm{C}_{3} \mathrm{H}_{5} \mathrm{O}_{3}\right)$ e dos diversos grupos acila (RCO)

4. A contribuição de cada resíduo na formação média do triglicerídeo corresponde ao \% RCOOH multiplicado por sua massa $\left(\mathrm{M}_{\mathrm{RCO}}\right)$.

5. A massa molar média do seu triglicerídeo é obtida pelo somatório 
do resíduo de glicerina adicionado do triplo da massa molar média dos grupos acila.

6. Utilize a tabela abaixo para realizar seus cálculos

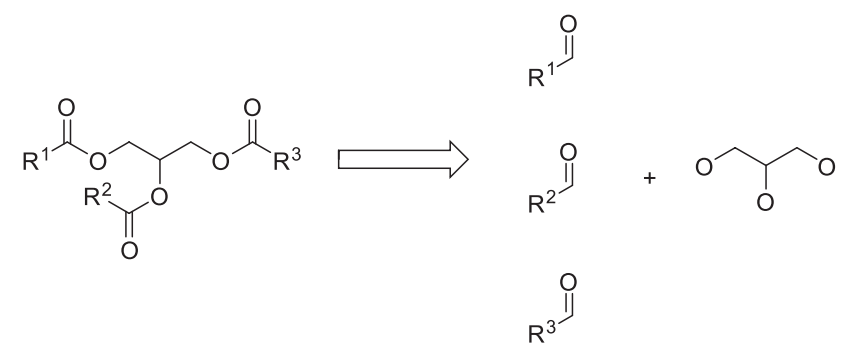

\begin{tabular}{|l|c|c|c|c|}
\hline Resíduo AG & Fórmula & $\begin{array}{c}\mathrm{M}_{\text {RCo }} \\
\left(\mathrm{g} \mathrm{mol}^{-1}\right)\end{array}$ & $\begin{array}{c}\% \\
\text { RCOOH }\end{array}$ & $\begin{array}{c}\text { Contri- } \\
\text { buição } \\
\left(\mathrm{g} \mathrm{mol}^{-1}\right)\end{array}$ \\
\hline Resíduo de glicerol & $\mathrm{C}_{3} \mathrm{H}_{5} \mathrm{O}_{3}$ & & - & \\
\hline Mirístico (C14:0) & $\mathrm{C}_{14} \mathrm{H}_{27} \mathrm{O}$ & & & \\
\hline Palmítico(16:0) & $\mathrm{C}_{16} \mathrm{H}_{31} \mathrm{O}$ & & & \\
\hline Esteárico(18:0) & $\mathrm{C}_{18} \mathrm{H}_{35} \mathrm{O}$ & & & \\
\hline Oleico(18:1) & $\mathrm{C}_{18} \mathrm{H}_{33} \mathrm{O}$ & & & \\
\hline Linoleico(18:2) & $\mathrm{C}_{18} \mathrm{H}_{31} \mathrm{O}$ & & & \\
\hline Linolênico (C18:3) & $\mathrm{C}_{18} \mathrm{H}_{29} \mathrm{O}$ & & & \\
\hline \multicolumn{7}{|l}{ Massa molar média do triglicerídeo $\left(\mathrm{g} \mathrm{mol}^{-1}\right)$} \\
\hline
\end{tabular}

\section{Notas ao professor}

\section{Lista de materiais}

\section{Determinação do índice de acidez (IA) do triglicerídeo}

Bureta $10 \mathrm{~mL}$; Erlenmeyer $250 \mathrm{~mL}$ - 4 unidades; etanol PA 150 $\mathrm{mL}$; solução de fenolftaleína; $\mathrm{NaOH}$ aq. $0,1 \mathrm{~mol} \mathrm{~L}^{-1}$ padronizado - 50 $\mathrm{mL}$; suporte universal e suporte para bureta; agitador magnético com chapa de aquecimento; magneto - 3 unidades; proveta de $50 \mathrm{~mL}$.

\section{Determinação da massa específica do triglicerídeo}

Balão volumétrico $10 \mathrm{~mL}-3$ unidades e pipeta Pasteur.

\section{Obtenção de biodiesel por transesterificação de um óleo: rota metílica}

Pyrex para banho-maria; suporte universal e garra para aparelhagem de refluxo; agitador magnético com chapa de aquecimento; magneto; termômetro (até $110{ }^{\circ} \mathrm{C}$ ); Béquer de $50 \mathrm{~mL}-4$ unidades; proveta de $50 \mathrm{~mL}$; bastão de vidro; espátula; $\mathrm{KOH} 1 \mathrm{~g}$; metanol PA 50; óleo vegetal $50 \mathrm{~mL}$; balão de fundo redondo de $125 \mathrm{~mL}(24 / 40)$ - 3 unidades; condensador de refluxo (24/40); mangueiras; pipeta 1 $\mathrm{mL}$; ácido sulfúrico concentrado $1 \mathrm{~mL}$; funil de Büchner, kitazzato e papel filtro; rotavapor e bomba de vácuo; funil de separação de 125 mL; argola para funil de separação.

\section{Determinação da massa específica do biodiesel}

Balão volumétrico $10 \mathrm{~mL}-3$ unidades; pipeta Pasteur.

\section{Determinação da viscosidade relativa do biodiesel}

Pipeta volumétrica de $5 \mathrm{~mL}$; haste universal e suporte para bureta; pera de sucção; Béquer de $50 \mathrm{~mL}-3$ unidades; heptano, $5 \mathrm{~mL}$.

\section{Cromatografia de camada delgada do biodiesel}

Eluente: heptano, éter etílico e ácido acético glacial (80:10:1), $5 \mathrm{~mL}$; cuba para cromatografia; capilares para cromatografia; cromatoplacas de silicagel; câmara de iodo; pinça; pipeta graduada de
$5 \mathrm{~mL}$; tubos de ensaio e estante para tubos; heptano; pipeta Pasteur -3 unidades; papel filtro.

\section{Determinação da pureza do biodiesel}

Preparação da solução padrão interno de heptadecanoato de metila ca $10 \mathrm{mg} \mathrm{mL}^{-1}$

Balão volumétrico de $25 \mathrm{~mL}, 1$ unidade; ca $250 \mathrm{mg}$ de heptadecanoato de metila (99\%); heptano, $25 \mathrm{~mL}$.

\section{Preparação da amostra}

Frasco de $10 \mathrm{~mL}, 3$ unidades; pipeta volumétrica $5 \mathrm{~mL}$.

\section{Análise cromatográfica}

A pureza do biodiesel foi determinada em um cromatógrafo gasoso GC 2010 Shimadzu equipado com detector por ionização em chama, injetor automático AOC 20i e coluna OV-Carbowax $20 \mathrm{M}(30 \mathrm{~m} \times 320 \mu \mathrm{m} \times 0,25 \mu \mathrm{m})$. A temperatura inicial de forno foi ajustada em $160{ }^{\circ} \mathrm{C}$ (2 min) com taxa de aquecimento de $4{ }^{\circ} \mathrm{C}$ $\min ^{-1}$, até a temperatura final de $230^{\circ} \mathrm{C}$. Um volume de $1,0 \mu \mathrm{L}$ foi injetado automaticamente no modo split (10:1). O gás de arraste utilizado foi He, com vazão de $2 \mathrm{~mL} \mathrm{~min}{ }^{-1}$. A integração deve ser ajustada de forma a incluir todo o intervalo compreendido entre os picos identificados como os ésteres metílicos dos ácidos margárico (C14:0) e nervônico (C24:1).

\section{Observações}

1. Metanol, éter etílico e heptano são líquidos inflamáveis e tóxicos. Ácido sulfúrico, ácido acético glacial, hidróxido de sódio, hidróxido de potássio e iodo são corrosivos. A reação de transesterificação deve ser realizada em capela.

2. Os óleos vegetais e a banha suína utilizados são comerciais e foram adquiridos em supermercado. $\mathrm{O}$ sebo bovino foi adquirido no mercado e submetido a aquecimento a $90{ }^{\circ} \mathrm{C}$ até a separação em duas fases. A fase líquida, o sebo, foi separada por filtração a quente.

3. Na determinação da viscosidade relativa, qualquer pipeta pode ser utilizada. É recomendável, entretanto, que o tempo de escoamento do biodiesel seja aproximadamente $15 \mathrm{~s}$ para minimizar o erro da medida. As viscosidades relativas dos biodieseis de sebo, banha, óleo de pequi e palma não foram determinados porque as matérias-primas são sólidas à temperatura ambiente.

4. Heptano pode ser substituído por hexano em qualquer experimento.

5. A Figura $1 \mathrm{~S}$ apresenta a cromatoplaca do experimento envolvendo óleo de canola. Neste, e também nos demais casos, a amostra de biodiesel não contém quantidades detectáveis de matéria-prima, indicando a alta conversão da reação e pureza do produto.

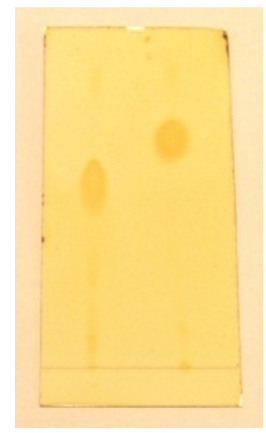

Figura 1S. Cromatografia de camada delgada da matéria-prima (esquerda) e do produto (direita) da reação de transesterificação do óleo de canola (eluído em hexano:éter etílico:HOAc 80:19:1, revelado em $I_{2}$ ) 
6. A reação pode ser acompanhada por CCD pela retirada de alíquotas em intervalos regulares.

7. A Figura $2 \mathrm{~S}$ apresenta os cromatogramas de três amostras típicas (biodieseis de óleos de arroz, milho e canola). Nas condições de análise, os principais componentes são eluídos na seguinte ordem: C16:0 (4,6 min), C18:0 (6,9 min), C18:1 (7,2 min), C18:2 (7,9 $\min )$ e $\mathrm{C} 18: 3(8,7 \mathrm{~min})$. O pico do padrão interno, $\mathrm{C} 17: 0$ foi observado em $5,7 \mathrm{~min}$.

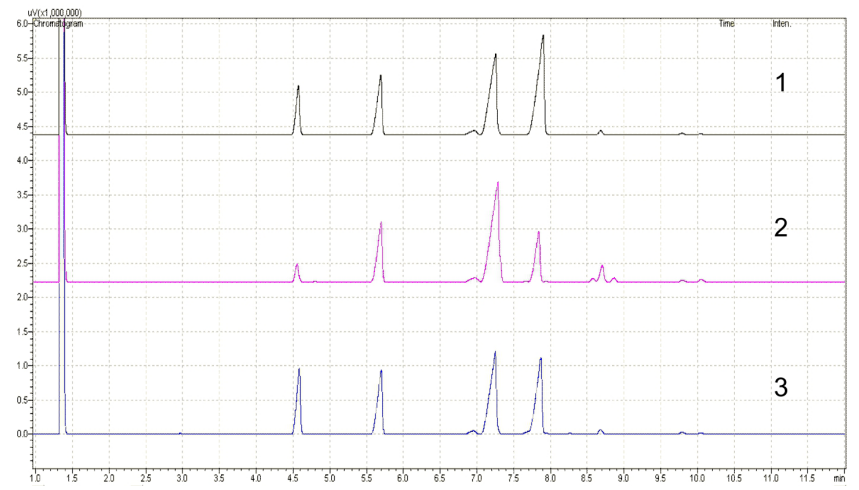

Figura 2S. Cromatogramas dos biodieseis de arroz (1), milho (2) e canola (3)
8. Biodieseis de sebo e banha apresentam quantidade significativa de heptadecanoato de metila (C17:0). Neste caso, soluções da amostra com e sem a adição do padrão interno são preparadas e analisadas. A Equação 6 deve ser utilizada para corrigir a área do padrão interno $\left(A_{P I C}\right)$.

$$
A_{P I C}=C_{A} \times\left[\frac{A_{P I A}}{C_{A}}-\frac{A_{P I B}}{C_{B}}\right]
$$

na qual, $A_{P I A}$ é a área do pico do C17:0 na amostra com adição de padrão interno; $A_{P I B}$ é a área do pico do C17:0 na amostra sem adição de padrão interno; $C_{A}$ é a concentração da solução da amostra com adição de padrão interno; $C_{B}$ é a concentração da solução da amostra sem adição de padrão interno.

9. A revisão de 2011 da norma EN14103 para a determinação do teor de ésteres graxos em biodiesel, entre outras modificações, substituiu o padrão heptadecanoato de metila pelo seu homólogo superior nonadecanoato de metila e ampliou a faixa de integração de C14-C24 para C6-C24.

10. A determinação do índice de iodo pode ser utilizada como forma de medida do teor de insaturações na composição do biodiesel e relacionada com o estado físico da matéria-prima. 